\title{
Estimate of Vascular Permeability and Cerebral Blood Volume Using Gd-DTPA Contrast Enhancement and Dynamic T2*-Weighted MRI
}

\author{
Yue Cao, PhD, ${ }^{1,2}$ Zhen Shen, MA, ${ }^{1}$ Thomas L. Chenevert, $\mathrm{PhD},{ }^{2}$ and \\ James R. Ewing, $\mathrm{PhD}^{3}$
}

\begin{abstract}
Purpose: To develop a numerical approach for estimation of vascular permeability from dynamic T2*-weighted imaging, a technique routinely used to measure cerebral blood volume (CBV) and flow in gliomas.

Materials and Methods: This study describes a process for estimating both the gadolinium diethylenetriamine pentaacetic acid (Gd-DTPA) transvascular transfer constant and CBV from dynamic $\mathrm{T} 2 *$-weighted images. The algorithm was applied to data from the brains of 12 patients with grade IV gliomas. The stability of the method was assessed. Estimates of CBV by this technique were compared to those of the conventional method.
\end{abstract}

Results: The algorithm was found to be insensitive to noise and to generate stable voxel-by-voxel estimates of permeability and CBV.

Conclusion: Using a single imaging acquisition, the three most important vascular properties, CBV, cerebral blood flow (CBF), and permeability, can be estimated. This approach may have potential in clinical evaluation of patients with brain tumor or acute ischemic stroke.

Key Words: DCE; vascular permeability; MRI; gliomas; cerebral blood volume

J. Magn. Reson. Imaging 2006;24:288-296.

(C) 2006 Wiley-Liss, Inc.

VASCULAR PERMEABILITY to a contrast agent is a potentially valuable measurement for evaluating cancer

${ }^{1}$ Department of Radiation Oncology, University of Michigan, Ann Arbor, Michigan, USA.

${ }^{2}$ Department of Radiology, University of Michigan, Ann Arbor, Michigan, USA.

${ }^{3}$ Department of Neurology, Henry Ford Health System, Detroit, Michigan, USA.

Contract grant sponsor: National Institutes of Health (NIH); Contract grant numbers: 2 PO1 CA59827, PO1 CA85878, RO1 HL70023, R21 CA1 1369901

*Address reprint requests to: $\mathrm{Y} . \mathrm{C} ., \mathrm{PhD}$, Department of Radiation Oncology, University of Michigan, 1500 E. Medical Center Drive, Rm. B2C438, Box 0010, Ann Arbor, MI 48109-0010.

E-mail: yuecao@med.umich.edu

Received June 13, 2005; Accepted April 17, 2006

DOI 10.1002/jmri.20634

Published online 22 June 2006 in Wiley InterScience (www.interscience. wiley.com) therapy efficacy and/or related normal tissue toxicity. Most pharmacokinetic modeling of vascular permeability is based upon dynamic contrast-enhanced (DCE) T1 MR measurements (1-3). Although some concerns remain in the estimation of contrast agent concentrations using DCE T1 measures (4-6), the methodologies for permeability quantification have been reviewed and a consensus has been reached (7). Interest in estimating vascular permeability to gadolinium diethylenetriamine pentaacetic acid (Gd-DTPA) from DCE T2*-weighted images has recently increased (8), driven primarily by clinical research interests in obtaining perfusion parameters of cerebral blood volume (CBV) and cerebral blood flow $(\mathrm{CBF})$, along with a measure of the vascular permeability estimate in a single bolus contrast injection. Single-shot T2*-weighted DCE is commonly utilized for whole-brain mapping of $\mathrm{CBV}$ and $\mathrm{CBF}$, especially in clinical settings, due to its high temporal resolution (one to two seconds) and tissue coverage; whereas T1sensitive DCE acquisition is desirable to measure permeability. Competing factors, however, make it difficult to accentuate both $\mathrm{T} 1$ and $\mathrm{T} 2 *$ contrast during the passage/leakage of an intravascular agent, so that typically only one contrast type is selected for a given injection. Ideally, two contrast injections are performed in separate series to independently determine permeability and blood volume/flow. This may add significant cost, scan time, and protocol complexity, and thus twoinjection MRI exams are usually not performed. As a potential practical alternative, estimation of CBV has been proposed from a T1-weighted DCE images series based on signal enhancement during the first pass of a bolus while it is still confined to the vasculature (9). This is not ideal, since T1-weighted techniques typically do not have the temporal and spatial resolution of T2* weighted echo-planar imaging (EPI). In another approach, both $\mathrm{T} 1$ - and $\mathrm{T} 2 *$-sensitive images are simultaneously acquired by dual gradient-echo dynamic imaging $(10,11)$, but this method requires a tradeoff between the temporal and spatial resolution. Conversely, contrast leakage into the extravascular space alters signal on $\mathrm{T} 2 *$-weighted images so that it may be possible to derive vascular permeability by analysis of 
agent recirculation and equilibration during the latter time points of a dynamic $\mathrm{T} 2 *$-weighted series (8). The objective of the work presented here was to investigate the performance of a new algorithm for deriving permeability information from a dynamic T2*-weighted image series. While quantification of multiple vascular properties from a single injection has substantial practical appeal, the stability and accuracy of such measures performed on a voxel-by-voxel basis is difficult to predict without clinical trials. Moreover, a hybrid approach is not anticipated to exceed the performance of dedicated (i.e., two-injection) methods, but the tradeoffs may be tolerable and permit retrospective estimation of additional useful properties.

The theoretical framework for correction and ultimate utilization of $\mathrm{T} 1$ enhancement in predominately $\mathrm{T} 2{ }^{*}$ weighted images has been described by others (12). Nevertheless, using T2*-weighted images to obtain stable and reproducible voxel-by-voxel estimates for blood-to-brain/tumor permeability to a MR contrast agent remains a challenge. A method proposed by Weisskoff et al (12), and modifications of that work $(13,14)$, were primarily designed to correct the influence of $\mathrm{T} 1$ enhancement on the estimate of CBV; the method was later used for the estimate of vascular permeability in a clinical application (15). The effect of T1 enhancement on the estimate of CBV, however, can be substantially reduced by using T2* instead of T2 measures, long TR, small flip angle, and relatively low contrast dose. Note that in Weisskoff's methods, back-flux of the contrast agent from extravascular extracellular space (EES) to the vascular space was neglected; at high values of permeability, this can cause estimates to be biased toward lower values (6). Several other methodologies have also been proposed to simultaneously quantify permeability and CBV from dual gradient-echo dynamic images $(10,11)$. Again, in these studies, the back-flux of the tracer was neglected. As a drawback, dual gradient-echo dynamic imaging has limited speed, so the investigators often trade spatial resolution or coverage (one to two slices) for temporal resolution ( 1-2 seconds per frame).

A recent methodology (8) has been presented in which the contrast concentration-time curve was determined from normal white matter, the back-flux of the tracer from the EES to the vascular space was considered, and the difference between intravascular and extravascular contrast-induced susceptibilities was neglected. This approach was able to qualitatively differentiate pathologies with different vascular properties. However, the technique is very sensitive to signal-to-noise and fails to produce voxel-by-voxel permeability or CBV corrected for vessel leakage.

Combining elements from these earlier works, we propose an iterative approach to estimate, voxel-byvoxel, the permeability map and CBV map from dynamic $\mathrm{T} 2 *$-weighted MR images. The algorithm is applied to estimate the transfer constant of Gd-DTPA and CBV in the brains of 12 patients with grade IV gliomas. The estimate of CBV by this technique is compared to that calculated by a conventional method (16). The sta- bility of the algorithm is assessed by simulated data. Results indicate the algorithm is insensitive to noise and can generate stable estimates of permeability and CBV on a voxel-by-voxel basis (17).

\section{MATERIALS AND METHODS}

\section{Theory}

The total tissue concentration of an extracellular contrast agent can be described as (7)

$$
\begin{aligned}
C_{t}(t)=v_{p} C_{p}(t)+v_{e e s} C_{e e s}(t) & =v_{p} C_{p} \\
& +K_{\text {trans }} \int_{0}^{t} C_{p}(\tau) \exp ^{-k_{e p}(t-\tau)} d \tau
\end{aligned}
$$

where $C$ denotes the tracer concentration, $v$ depicts the fractional volume, subscripts $t, p$, and ees represent respectively tissue, plasma, and EES, $K_{\text {trans }}$ is the transfer constant of the agent influx from the plasma to the $\mathrm{EES}$, and $k_{e p}$ is the tracer efflux rate from the EES to the plasma. The fractional volume of the EES can be determined from the ratio of $K_{\text {trans }}$ to $k_{e p}$.

\section{Bolus Shape Function}

The first-pass bolus shape function has been described by a gamma variate $g(t)$ plus an integral term $(8,12)$ :

$$
s_{\text {inp }}(t)=A\left[g(t)+\lambda \int_{0}^{t} g(t-\tau) d \tau\right] \text {, }
$$

where

$$
\begin{aligned}
& g(t)=\left(t-t_{0}\right)^{\alpha} \exp \left(-\beta\left(t-t_{0}\right)\right) \text { for } \\
& t>t_{0}, \text { otherwise } g(t)=0 .
\end{aligned}
$$

In most proposals $(8,10-14)$, the recirculation "bump" in the bolus shape function has been neglected. However, in almost all cases we have examined, the second pass closely follows the first pass (Fig. 1). Neglecting the second pass can result in substantial errors in estimates of $K_{\text {trans }}$ (Fig. 1d). Thus, an additional term that describes the second pass was added to the bolus shape function (Eq. [2]) as

$$
s_{\text {inp }}^{2 n d}=A^{\prime}\left[\left(t-t_{0}^{\prime}\right) / t_{\text {half }}\right]^{\alpha} \exp \left(-\beta\left(t-t_{0}^{\prime}\right) / t_{\text {half }}\right),
$$

where $t_{0}^{\prime}, t_{\text {half }}$, and $A^{\prime}$ are parameters adjusted for the time shift, width, and amplitude, respectively, of the bolus' second pass.

\section{Algorithm}

Our fitting process consists of five steps:

1. Assume $K_{\text {trans }}$ is zero and $v_{p}$ is $1 \%$ in normal white matter $(18,19)$. Thus, Eq. [1] becomes:

$$
C^{w m}(t)=v_{p}^{w m} s_{i n p}^{w m}(t) .
$$



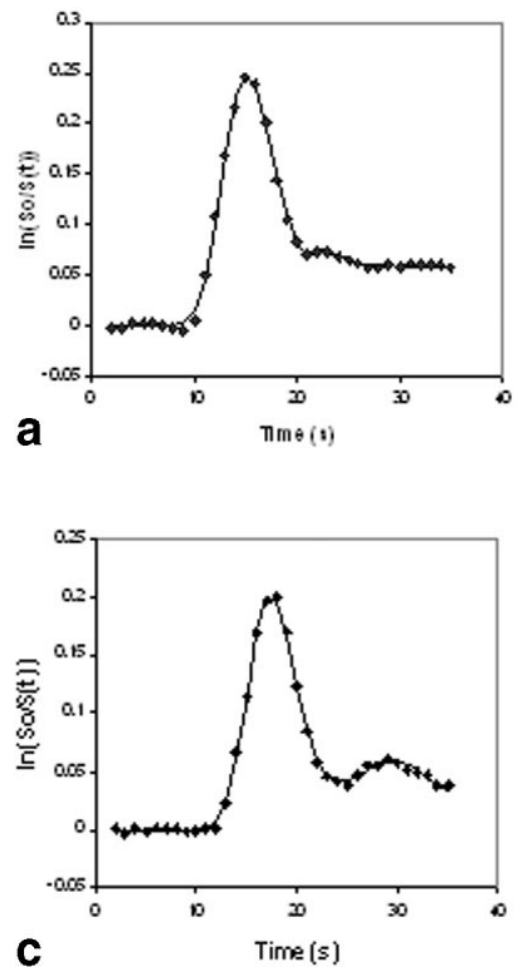
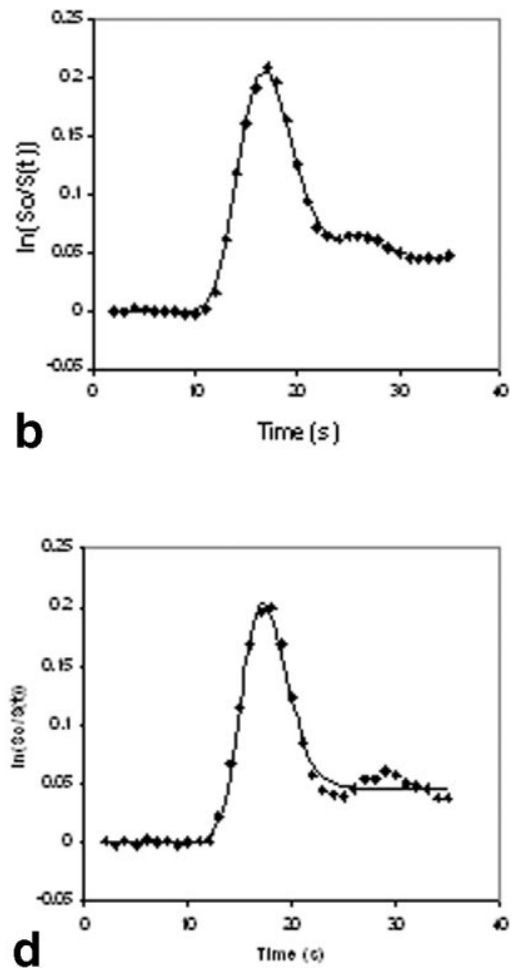

Figure 1. Contrast concentration-time curves in normal white matter of three patients $(\mathbf{a}-\mathbf{c})$. Note that the recirculation of the contrast bolus follows the first pass very closely. The intensity of the recirculation "bump" is significantly greater than that in the tail (d). For various shapes of the contrast concentration-time curves, accurate fits (solid lines) can be obtained from Eqs. [2]-[4]. If only the first pass is considered, the residual sum of squares of errors (d) can be an almost order magnitude greater than that of fitting both the first- and second-bolus passes (c).
Fitting Eq. [5] to the data in volumes of interest (VOIs) of normal white matter yields eight parameters $\left(\alpha, \beta, \lambda t_{0}\right.$, and $A$ for the first pass, and $t_{0}{ }^{\prime}, t_{\text {half }}$, and $A^{\prime}$ for the second pass) that describe the bolus shape function.

2. For the first estimate of $v_{p}$ in each voxel, a rapid increase in $C_{t}$ up to the peak of the first pass is assumed to be predominately due to contrast agent in the intravascular space. This assumption is consistent with the computation of the CBV by the conventional algorithm (16). In addition, a possible time shift of the input function in a voxel is considered and quantified compared to that in normal white matter. However, the change in the width of the input function is omitted due to the fact that there is insufficient information to deter-

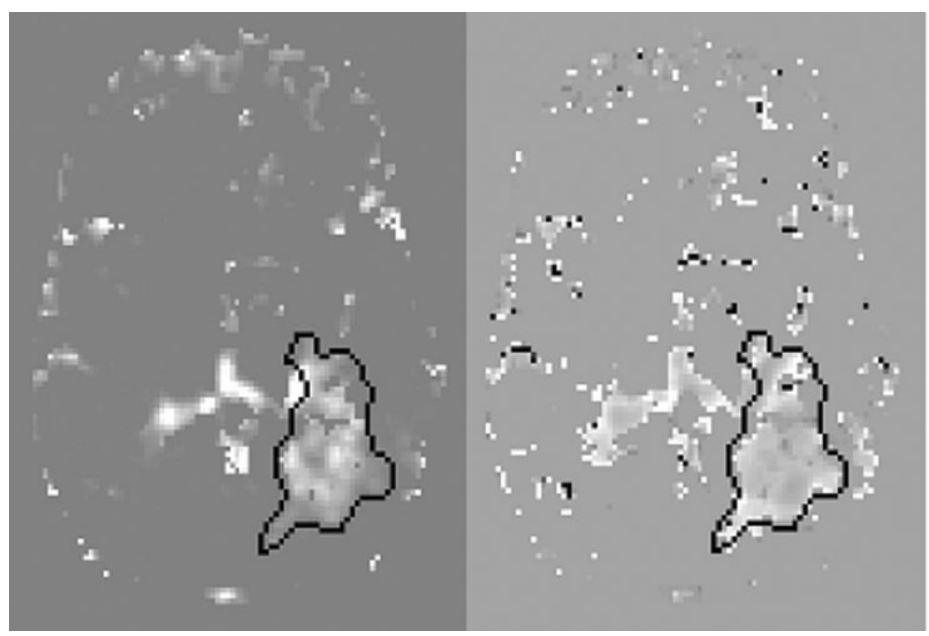

mine it. With these assumptions, the input function for each voxel can be written as:

$$
s_{i n p}^{\text {vox }}(t)=s_{i n p}^{w m}\left(t-t_{0}^{v o x}\right)
$$

Fitting the temporal data up to the peak of the first pass in a voxel using Eq. [6] yields an estimate of the parameters $v_{p}^{\text {vox(1) }}$ and $t_{0}^{\text {vox }}$.

3. Before computing $K_{\text {trans }}$, the presence of significant vascular leakage in each voxel is assessed. The difference of the total contrast concentration in a voxel and the contribution of the vascular volume to the concentration in the voxel (see Fig. 2) is determined by

Figure 2. a: Shows the transfer constant map estimated by fitting both the first and second passes of the bolus in the input function. b: Shows the difference between the transfer constant maps estimated by fitting both the first and second passes of the bolus and neglecting the recirculation; positive values are shown in the tumor volume (black contour), suggesting that neglecting the recirculation yields an underestimate of the transfer constant. 


$$
\begin{aligned}
C_{t}^{\text {vox }}(t)-v_{p}^{\text {vox }(1)} s_{\text {inp }}^{\text {vox }}(t)= & K_{\text {trans }} \int_{0}^{t} \\
& s_{\text {inp }}^{\text {vox }}(\tau) \exp \left(-k_{e p}(t-\tau)\right) d \tau
\end{aligned}
$$

If Eq. [7] is significantly greater than zero, it indicates that $K_{\text {trans }}$ is detectable (discernable from noise). Otherwise, fitting $K_{\text {trans }}$ can cause unstable results and errors in both $K_{\text {trans }}$ and $v_{p}$. Thus, a $t$-test is used to evaluate Eq. [7]. The mean and standard deviation (SD) of the different signals in Eq. [7] from the time at the peak of the first pass of the bolus to the end of the time curve are calculated. Then, a $t$-value is calculated as the ratio of the mean to the standard error of the mean (SEM). If the $t$-value is significantly greater than zero (onetailed $t$-test; $P<0.05$ ), the computation continues for step 4 , and if not, $K_{\text {trans }}$ and $k_{e p}$ are assigned to be zero and the program stops.

4. Fit Eq. [7] for the second half of the curve (starting from the peak) to obtain the first estimates of $K_{\text {trans }}^{(1)}$ and $k_{e p}^{(1)}$ while keeping $v_{p}^{\operatorname{vox}(1)}$ and $t_{0}^{\text {wox }}$ fixed.

5 . Refit the whole curve for $K_{\text {trans }}, K_{e p}$, and $v_{p}$ by allowing perturbations of the values obtained from steps 2 and 4 with fixed $t_{0}^{\text {wox }}$.

\section{MRI Experiment and Subjects}

Retrospective analysis was performed on dynamic T2*weighted images acquired in 12 patients with grade IV glioma participating in a clinical protocol approved by the internal review board. The images were acquired one to two weeks prior to radiotherapy, but two to four weeks after surgery (either partial resection or biopsy only). Informed consent was obtained from all of the patients prior to the study.

All images were acquired on a 1.5-Tesla clinical MRI scanner (General Electric Medical Systems, Milwaukee, WI, USA). A total of 36 volumes of dynamic T2*weighted images were acquired by a gradient-echo EPI pulse sequence with $\mathrm{TR}=2$ seconds, $\mathrm{TE}=60 \mathrm{msec}$, field-of-view $=220 \times 220 \mathrm{~mm}^{2}$, matrix size $=128 \times$ 128 , flip angle $=60^{\circ}$, and 14 interleaved slices with thickness $=6 \mathrm{~mm}$ and gap $=0 \mathrm{~mm}$. Gd-DTPA $(0.1$ $\mathrm{mL} / \mathrm{kg}$ ) was injected intravenously by a power injector with a rate of $2 \mathrm{~mL} /$ second, followed immediately by 15 $\mathrm{mL}$ of saline flush at the same rate.

The paramagnetic contrast agent that has been previously used for estimates of CBV and CBF $(16,20,21)$ gives rise to a change in $R_{2}{ }^{*}$, which has been (either implicitly or explicitly) assumed to be proportional to the intravascular contrast concentration $(16,20,22)$ as

$$
\Delta R_{2 \text { intra }}^{*}=\gamma_{\text {intra }} C_{p}
$$

where $\gamma_{\text {intra }}$ is the proportional factor, and $C_{p}$ is the plasma contrast concentration. The effective $R_{2}$ * change in a voxel that contains both intravascular and extravascular contrast agents can be approximated as

$$
\Delta R_{2 e f f}^{*}=\gamma_{e f f} C_{t}
$$

where $\gamma_{\text {eff }}$ is the effective proportional factor, and $C_{t}$ is the total contrast concentration in tissue. Given that the $R_{2}$ * effect depends upon the size of the contrast distribution space (23), $\gamma_{\text {intra }}$ and $\gamma_{\text {eff }}$ may be different, and given the difficulty of constructing model systems representative of capillary vascular beds, not easily determined by in vitro studies. Using Eq. [1], [8], and [9], the effective $R_{2}{ }^{*}$ change in a voxel can be expressed as

$$
\begin{aligned}
& \Delta R_{2 \text { eff }}^{*}=\frac{\gamma_{\text {eff }}}{\gamma_{\text {intra }}}\left[v_{p} \Delta R_{2 \text { intra }}^{*}(t)+K_{\text {trans }} \int_{0}^{t}\right. \\
& \left.\Delta R_{2 \text { intra }}^{*}(\tau) \exp \left(-k_{e p}(t-\tau 0)\right) d \tau\right]=\left[v_{p}^{\prime} \Delta R_{2 \text { intra }}^{*}(t)+K_{\text {trans }}^{\prime} \int_{0}^{t}\right. \\
& \left.\Delta R_{2 \text { intra }}^{*}(\tau) \exp \left(-k_{e p}(t-\tau 0)\right) d \tau\right],
\end{aligned}
$$

where Eq. [10] is similar to Eq. [1], except that $v_{p}^{\prime}$ and $K^{\prime}{ }_{\text {trans }}$ are scaled by the ratio of $\gamma_{\text {eff }}$ to $\gamma_{\text {intra }}$. For a voxel that contains no leaky vessels, the ratio is equal to one. The $\Delta R_{2} *$ is obtained from the EPI sequence described previously as

$$
\Delta R_{2}^{*}=-\frac{1}{T E} \ln \left(\frac{S}{S_{o}}\right)
$$

where $S_{o}$ and $S$ are the signal intensities acquired before and after the contrast bolus injection, respectively.

\section{Numerical Computation}

All software programs were developed in-house using $\mathrm{C}++$. Prior to computations for $K_{\text {trans }}^{\prime}$ and $v_{p}^{\prime}$, acquisition time differences between sections within a single volume were corrected by cubic spline interpolation (24) to match the time of the first slice acquired in each volume. All of the fitting described in the Theory section was done by minimizing the residual sum of squares of errors using a simplex search algorithm (24).

To estimate the parameters (up to eight) of the input function, a typical VOI of normal white matter was selected, containing 500-800 voxels that had a contrast-noise ratio (CNR) of 100-150. CNR is defined as the difference in signal intensities between the peak of the first pass of the bolus and the baseline average divided by the SD of the baseline signal intensities. Several guidelines were used for selection of the white matter VOI: 1) normal appearance in T2-weighted images; 2) contralateral to the tumor; 3) as large as possible but avoiding partial volume averaging with gray matter and cerebrospinal fluid (CSF); and 4) avoiding regions degraded by susceptibility artifact. Also, voxels that had signal intensities 2SD above or below the mean were rejected from the VOI. To obtain the eight parameters of the input function, the residual sum of squares of errors from fitting the white matter VOI was minimized iteratively until the relative change between two subsequent iterations was less than $1 \times 10^{-8}$, a process which took about six to eight seconds. A similar iterative procedure was also applied to fit individual voxels, for which two parameters were fitted in step 2 

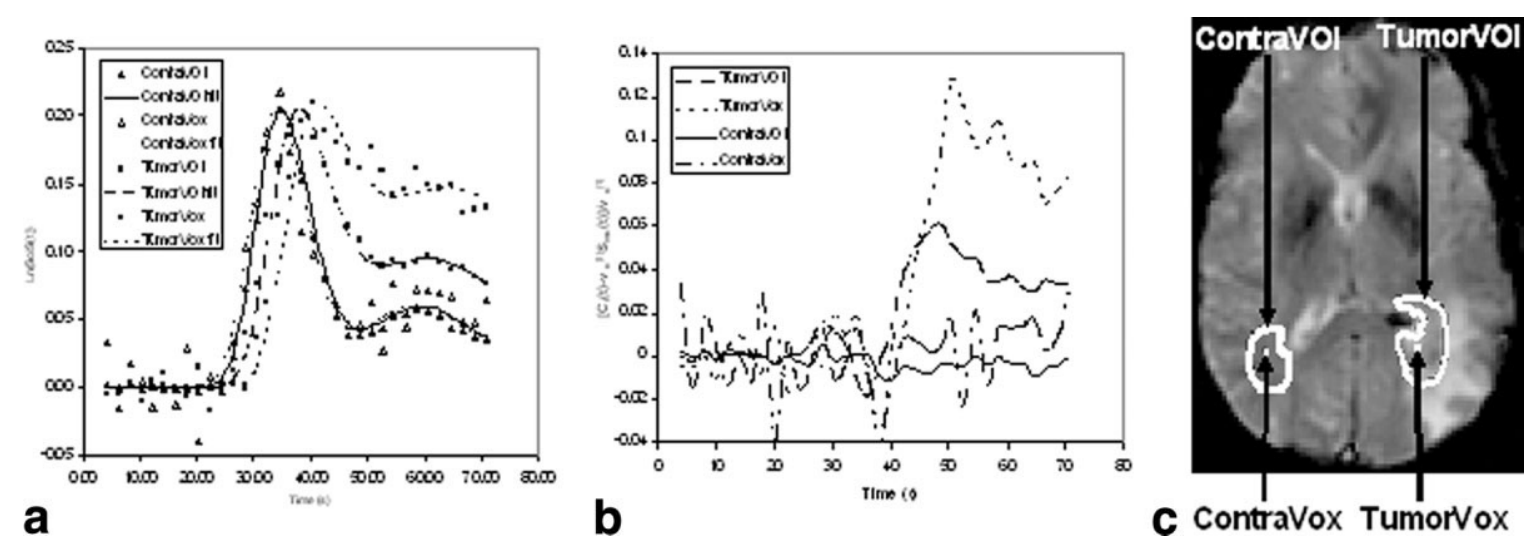

Figure 3. a: Shows contrast concentration-time curves in a VOI (TumorVOI) and a voxel (TumorVox) in the tumor region and a VOI (ContraVOI) and a voxel (ContraVox) in the region contralateral to tumor normalized by their fractional volumes of CBV. The solid, dotted, dash, and dotted-dash lines show the fitting results. b: Plots show the differences of a total of the contrast concentrations and the intravascular contributions to the concentration, presumably the extravascular tracer concentrations described by Eq. [7], in the two VOIs and two voxels. c: Shows the locations of the two VOIs and the two voxels, and arrows point to the voxels. Note that only the differences from the VOI and the voxel in the tumor region are significantly greater than zero $(P<$ 0.05), whereas those from the region contralateral to tumor are not.

$\left(\begin{array}{l}\text { vox(l) } \\ p\end{array}\right.$ parameters in step $5\left(K_{\text {trans }}, k_{e p}\right.$, and $\left.v_{p}\right)$.

\section{Stability Test}

The stability of the fitting algorithm is crucial for its usefulness in a clinical trial. Thus, the stability was tested by computer simulated data, in which $v_{p}$ was $1 \%$, $K_{\text {trans }}$ varied from 0.001 to 0.5 minute $^{-1}$, and $v_{\text {ees }}$ was $15 \%$. For the clinical data obtained, the single-voxel CNR in the tumor varied from 18 to 50 . Thus, Gaussian noise was added to the simulated contrast concentration-time curves to have CNR of 20,50, 100, and infinite at the peak of the first passage of the bolus. The simulated contrast concentration-time curve contained 36 points with two seconds per point, which matched the clinical data that we obtained. For each combination of the tested parameters and noise levels, 4096 simulations were conducted. The SD of the 4096 simulations per condition was computed and was used as a metric to assess the stability of the algorithm.

\section{RESULTS}

Contrast concentration-time curves in the normal white matter of three patients, and their fitting results using Eqs. [2]-[4], are shown in Fig. 1. If the recirculation pass is ignored, there is substantial discrepancy between the fitting result and the empirical data (Fig. 1d). For the example shown in Fig. 1c and d, the residual sum of squares of errors obtained by fitting the first pass only was $1.1 \times 10^{-3}$, almost an order of magnitude greater than the value of $1.4 \times 10^{-4}$ that was obtained by fitting both the first and second passes (Fig. 1c). This error affects the estimate of $K_{\text {trans. }}^{\prime}$. The $K_{\text {trans }}^{\prime}$ value obtained by fitting both the first and second passes of the bolus was approximately $22 \%$ greater than that obtained by fitting the first pass only (Fig. 2); while estimates of $v_{p}^{\prime}$ obtained by the two fittings were very close $(<3 \%$ in difference), as expected. For the case shown in Fig. 2, the mean $K_{\text {trans }}^{\prime}$ in the tumor volume defined on the fluid attenuation inversion recovery (FLAIR) images were 0.028 and 0.023 minute $^{-1}$ with and without fitting the recirculation "bump," respectively.

In our algorithm, after the initial estimates of $v_{p}$ and $t_{0}^{\text {vox }}$ for an individual voxel or VOI (step 2), the difference between a total of the contrast concentration and the intravascular concentration described by Eq. [7] was evaluated to determine whether there was a significant extravascular contrast concentration, defined as the area under the temporal curve after the first-pass peak significantly differing from zero (Fig. 3b). The extravascular contrast concentration-time curves from a VOI and a voxel in the tumor volume were significantly greater than zero $(P<0.05)$, while the data from another VOI and a voxel in the region contralateral to tumor were not $(P>0.05)$. For the latter VOI and voxel, $K_{\text {trans }}^{\prime}$ was assigned to be zero. For the first VOI and voxel, the fitting procedure continued to steps 4 and 5 . The final fitting results are shown in Figs. 4 and 5.

An example of $K_{\text {trans }}^{\prime}$ and $v_{p}^{\prime}(\mathrm{CBV})$ maps in a patient with grade IV glioma reconstructed by our algorithm is shown in Fig. 4. Note that only the rim of the tumor has significant nonzero $K_{\text {trans }}^{\prime}$. CBV maps reconstructed by our algorithm are similar to the CBV estimated by the conventional method without correction for vascular leakage (Fig. 5) (16). A scatter-plot of the two CBV maps shown in Fig. 5c has a correlation coefficient of 0.83 , slope of 0.86 , and intercept of $0.27 \%$. As expected, the relative differences of the two CBV maps are similar to the $K_{\text {trans }}^{\prime}$ maps (Fig. 5). Particularly, in the tumor region, the CBVs estimated by our algorithm are smaller than those obtained by the conventional method; in the latter, extravascular contrast agent, is at least partially accounted for as intravascular contrast agent.

In the 12 patients with grade IV gliomas, the averaged $K_{\text {trans }}^{\prime}$ over the tumor volume that was defined on FLAIR images but excluding voxels having zero $K_{\text {trans }}^{\prime}$ 

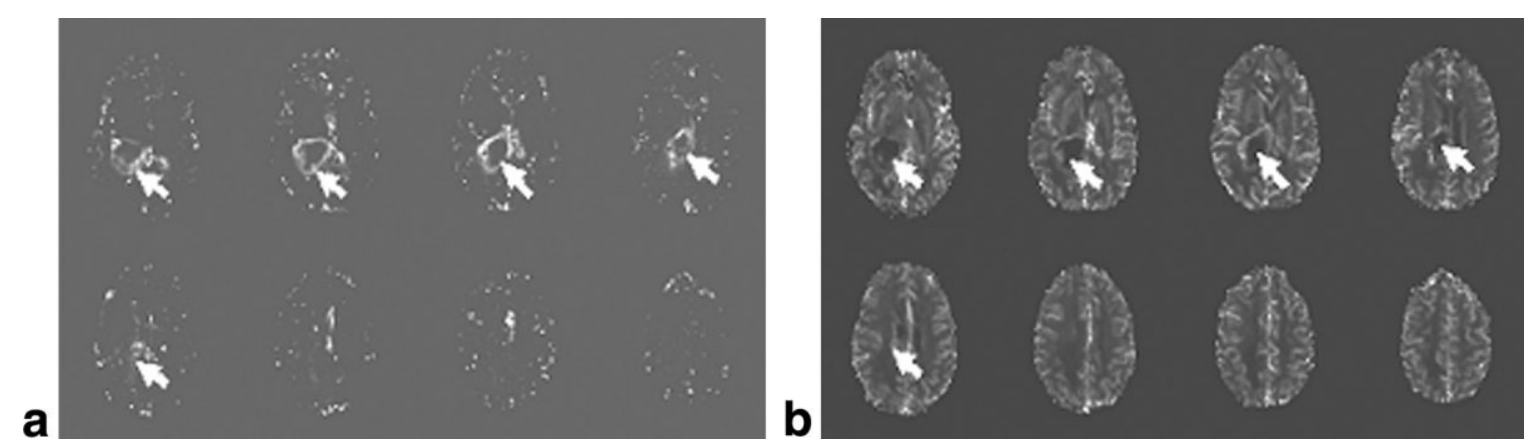

Figure 4. Maps of the transfer constants of Gd-DTPA (left panel) and the fractional volume of plasma (right panel) reconstructed by our algorithm. Note that only the rim of the tumor (arrowheads) in this patient shows an increase in the blood-brain/tumorbarrier permeability.

$\left(<0.0002\right.$ minute $\left.^{-1}\right)$ ranged from 0.12 minute $^{-1}$ to 0.02 minute $^{-1}$, the group mean of 0.07 minute $^{-1} \pm 0.03$ minute ${ }^{-1}$. Similarly, the average $v_{p}^{\prime}$ over the tumor volume ranged from $4.7 \%$ to $1.7 \%$, with a group mean of $2.7 \% \pm 0.9 \%$ (Table 1 ). SDs of $K_{\text {trans }}^{\prime}$ in individual tumors are also given in Table 1 , and are on the order of magnitude of $K_{\text {trans }}^{\prime}$ itself, suggesting heterogeneous vascular leakage in the tumor volume.

The stability of the algorithm was tested by computer simulated data. For $K_{\text {trans }}$ of 0.5 to 0.02 minute $^{-1}$ and four different CNRs, the ratio of the SD to the mean was less than $7 \%$. For $K_{\text {trans }}$ of 0.01 minute $^{-1}$, the ratio was less than $11 \%$, while for 0.005 minute $^{-1}$, the ratio was less than $33 \%$. For $K_{\text {trans }}$ of 0.002 and 0.001 minute $^{-1}$, the ratio was less than $33 \%$ for CNR greater than or equal to 50 and was greater than $100 \%$ for CNR of 20 (Fig. 6).

\section{DISCUSSION}

Accurately and consistently fitting the shape of the input function from normal white matter is crucial to minimize the error in voxel-by-voxel estimates of the transfer constant based upon dynamic T2*-weighted images. As demonstrated in Fig. 1, our algorithm eliminates the error associated with second-pass contrast recirculation. Successfully fitting the contrast agent recirculation reduced the residual sum of squares of er- rors by almost an order of magnitude compared to neglecting the recirculation. This error can greatly impact the estimate of the transfer constant, which is approximately $22 \%$ underestimated, as evidenced in Fig. 2. In order to fit the second pass, three additional parameters $\left(A^{\prime}, t_{0}{ }^{\prime}\right.$, and $\left.t_{\text {hal }}\right)$ were introduced to describe the width, height, and onset time of the second pass. Although there are up to eight parameters involved in fitting the input function from white matter, the CNR (approximately 150 at the peak of the curve) of the normal white matter curve obtained from a VOI that typically contains 500-800 voxels is sufficient for fitting. If in the case that there is no recirculation bump (we saw one such case out of 12 patients), $A^{\prime}$ (the amplitude of the recirculation bump) can be either manually or automatically set to zero. The ability to detect these eight parameters, although they are independent, can be influenced by the temporal resolution and the signal-to-noise ratio of the input function. However, it is worthwhile to point out that the accuracy in the fitting of the shape of the input function, determined by the mean squares error, affects the subsequent estimates of the transfer constant and the blood volume, but the precision of the individual parameters of the input function do not. Also, when the eight parameters are fitted, the shape of the input function is determined and is not altered (expect a possible time-shift) in the fitting for the transfer constant and the blood volume.
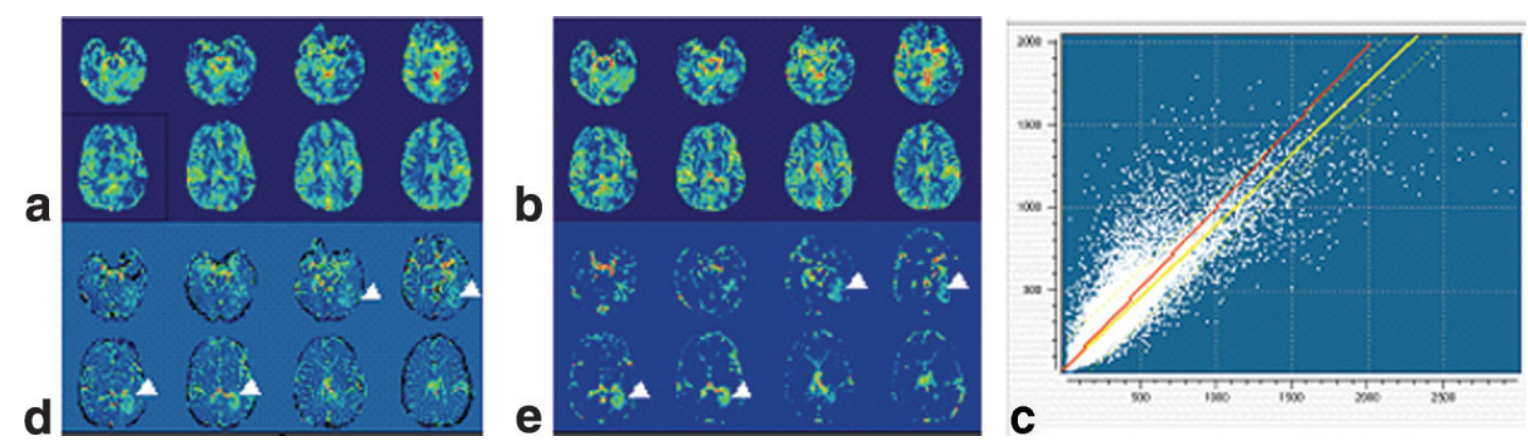

Figure 5. Two CBV maps of a patient with grade IV glioma with one reconstructed by our algorithm (a) and another by the conventional method (b). The two maps show the significant correlation (c), the yellow line is the linear regression, and the red line is the 45 degree line. The difference map (d) of the two CBV is similar to the transfer constant map (e), especially in the tumor region where the transfer constants are not zero (arrowheads). The CBV estimated by our algorithm is corrected for vascular leakage, and thus is smaller than that by the conventional method. 
Table 1

$K_{\text {trans }}^{\prime}$ and $v_{p}^{\prime}$ in Tumor Volumes of 12 Patients With Grade IV Gliomas

\begin{tabular}{lcccc}
\hline Patient \# & $\nu_{p}^{\prime}(\%)$ & SD & $\begin{array}{c}K_{\text {trans }}^{\prime} \\
\left(\text { minute }^{-1}\right)\end{array}$ & SD \\
\hline 1 & 1.7 & 1.3 & 0.045 & 0.052 \\
2 & 2.2 & 2.3 & 0.059 & 0.064 \\
3 & 1.9 & 1.4 & 0.021 & 0.030 \\
4 & 1.9 & 1.3 & 0.059 & 0.057 \\
5 & 2.7 & 1.7 & 0.037 & 0.052 \\
6 & 2.9 & 1.7 & 0.068 & 0.067 \\
7 & 2.5 & 1.8 & 0.120 & 0.150 \\
8 & 2.2 & 3.9 & 0.048 & 0.081 \\
9 & 4.2 & 4.6 & 0.112 & 0.150 \\
10 & 1.9 & 1.6 & 0.070 & 0.120 \\
11 & 4.7 & 3.7 & 0.084 & 0.120 \\
12 & 3.0 & 2.9 & 0.065 & 0.083 \\
Mean \pm SD & 2.7 & 1.0 & 0.066 & 0.029 \\
\hline
\end{tabular}

Given the single-voxel CNR of 18-50 (at the peak of the curve) that typically can be achieved in dynamic $\mathrm{T} 2 *$-weighted images on a 1.5-T scanner, a few steps in our algorithm are crucial to obtaining stable voxel-byvoxel estimates of the transfer constant and CBV. First, the difference in the width of the input function in a voxel from that in normal white matter is omitted. This omission is due to several considerations: 1) limited CNR; 2) limited temporal resolution; and 3) a broadening in the width of the contrast concentration-time curve in a voxel could also possibly be caused by vascular leakage (Fig. 3a). Thus, if the width of the input function for each voxel is allowed to vary freely, instability in fitting might result. On the other hand, the time shift of the input function in each voxel can be determined relatively easily from the initial, rapidly rising part of the curve, and there is no other parameter competing for this information (Fig. 3). Thus, the time-shift is adjusted for each voxel. Second, the initial voxel-byvoxel estimate of $v_{p}$ (step two) is on principle similar to the conventional CBV estimation when there is no vascular leakage and no width change of the input function in the voxel (16). The second part of the curve (after the peak of the first pass), including the broadening and elevation of the curve, is used for the initial estimates of $K_{\text {trans }}^{\prime}$ and $k_{e p}$. Third, an iterative process is used to readjust $v_{p}^{\prime}, K_{\text {trans }}^{\prime}$ and $k_{e p}$, since for a voxel that contains leaky vessels $v_{p}^{\prime}$ is potentially overestimated in step 2 and subsequently $K_{\text {trans }}^{\prime}$ is underestimated in step 4.

CBV as estimated by our algorithm is well correlated with that obtained from the conventional method (Fig. 5). The major differences between the two CBV maps are located in the regions with tumor and/or large vessels (Fig. 5d). Interestingly, but as expected, the pattern of the difference of the two CBV maps qualitatively resembles that of the transfer constant (Fig. 5e). In the brain and/or tumor regions where blood-brain/tumor barriers are disrupted and there is tissue uptake of the contrast agent, the contrast concentration-time curves are in general broadened and elevated (see Fig. 3a), which have been assigned partially if not completely to be a part of $\mathrm{CBV}$ by the conventional method. Our algorithm attributes the broadened and elevated contrast concentration-time curves in tissue (compared to that in normal white matter) to vessel leakage, and consequently the transfer constant of the contrast agent is estimated.

The averaged transfer constants of Gd-DTPA in the grade IV glioma volumes ranged from 0.02 to 0.12 minute $^{-1}$ with a group mean of 0.07 minute $^{-1}$, which is in the range of the values reported in the literature. In a group of 10 patients with glioblastoma multiforme, Johnson et al (8) found a mean transfer constant of 0.09 minute $^{-1}$ using dynamic T2*-weighted images and fitting the first pass only in the regions of interest (ROIs). In that study, the transfer constants were estimated within selected ROIs that showed the greatest signal changes during the bolus arrival; whereas in our study the transfer constants that were estimated voxelby-voxel were averaged over the voxels having nonzero transfer constants $\left(>0.0002\right.$ minute $\left.^{-1}\right)$ but within the tumor volume. The heterogeneity of the transfer constants in grade IV gliomas is well presented in Table 1, in which the SD is almost as great as the mean for each of the tumors. In 22 patients with gliomas, Roberts et al (25) reported the significant correlation between grades determined histologically and Gd-DTPA transfer constants estimated by using a T1-based approach and selecting the ROIs. If only grade IV gliomas in Robert et al's (25) study are considered, the averaged transfer constant was 0.094 minute $^{-1}(N=6)$, ranging from 0.4 to 0.13 minute $^{-1}$, essentially in agreement with our estimates. Using a modified T1 approach, Li et al (9) estimated that transfer constants ranged from 0.07 to 0.01 minute $^{-1}$, which were relatively smaller those reported by Roberts et al (25), Johnson et al (8), and us. Based upon our simulated data, the SD in estimates of the transfer constant increased with noise and with a decreased of the transfer constant (Fig. 6). For the 12 patients, the smallest averaged transfer constant in the FLAIR tumor volume is 0.021 minute $^{-1}$. For the transfer constant $\geqq 0.02$ minute $^{-1}$, our simulated data suggest that the ratio of the $\mathrm{SD}$ to the mean of the transfer

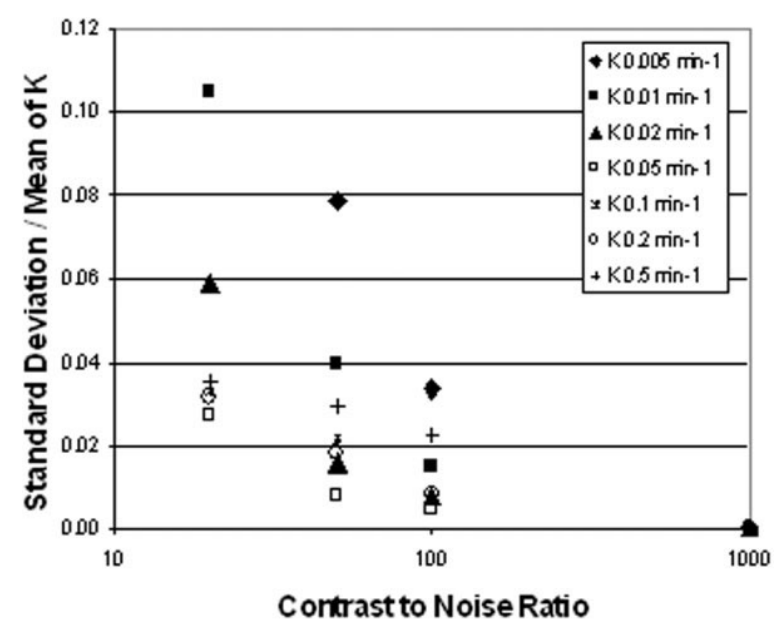

Figure 6. The ratio of the $\mathrm{SD}$ to the mean of the transfer constant as a function of the contrast to noise ratio at the peak of the first pass of the bolus. 
constant is less than 6\%. This indicates that the heterogeneity of the transfer constant in the tumor volume is not predominantly due to noise.

It is difficult to differentiate susceptibility induced by extravascular contrast from that arising form intravascular paramagnetic contrast (26). The relationship between the contrast concentration and induced susceptibility is complex, and may be influenced by the contrast distribution. Therefore, in vitro calibration is difficult to apply to in vivo data. The contrast agents distributed in the extravascular extracellular space most likely induce a $\mathrm{T} 2 *$ effect smaller than the contrast contained in microvasculature (23). As a result, neglecting this difference between intravascular and extravascular contrast-induced susceptibilities may cause an underestimation of the transfer constant.

In our computation, T1 enhancement (12) is not taken into account. Our data were acquired with long TR (two seconds), long TE (60 msec), 60 $0^{\circ} \mathrm{RF}$ pulse, and low contrast dose, and the signals never became negative within the 72 seconds acquisition time. Thus, T1 enhancement is a small effect, and most likely negligible. In step 3 of our algorithm, the contrast concentration-time curve in each voxel was compared to that in normal white matter after normalization by the first estimate of CBV. Only the voxels that show significant broadening and elevation after the peak of the first pass of the bolus compared to that in normal white matter are considered to have nonzero leakage. This step avoids fitting the transfer constant in the voxels where the contrast concentration-time curve after subtracting the intravascular contribution exhibits more noise than signal (see Fig. 3b), since such fitting can result in large errors in both the estimates of the CBV and the transfer constant and can cause instability.

The proposed algorithm shows excellent stability. For the single-voxel CNR of 20 or better that is routinely obtained in clinic, our simulation shows that the ratio of the SD to the mean is $11 \%$ or better for the transfer constant greater than or equal to 0.01 minute $^{-1}$. For the transfer constant of 0.005 minute $^{-1}$, the ratio increases to $33 \%$ for CNR of 20 . This latter increase in the SD of simulation reflects the detectability for small transfer constants using the current image acquisition parameters rather than the stability of the algorithm. The detectability can be improved by increasing data acquisition time to allow the contrast to infuse into tissue without an increase in CNR. For example, if the acquisition time is increased by 20 seconds, the ratio of the $\mathrm{SD}$ to the mean decreases from $33 \%$ to $14 \%$ for the transfer constant of 0.005 minute $^{-1}$ and CNR of 20.

In conclusion, the excellent stability of our algorithm is essential for using vascular permeability as a metric to evaluate a therapeutic regime (e.g., antiangiogenesis and antitumor) in a clinical trial. This algorithm allows us to retrospectively estimate the transfer constant of vasculature to Gd-DTPA from the dynamic T2*weighted images that are widely used for estimates of $\mathrm{CBV}$ and $\mathrm{CBF}$ in brain tumor clinic trials. Using a single image acquisition, the three most important vascular properties, CBV, CBF, and permeability, can be esti- mated, thus avoiding two contrast injections. Our approach may also have potential in clinical evaluation of patients with acute ischemic stroke, in which patient population a blood-brain-barrier (BBB) permeability increase can be an indicator of acute hemorrhage.

\section{REFERENCES}

1. Tofts PS, Kermode AG. Blood brain barrier permeability in multiple sclerosis using labelled DTPA with PET, CT and MRI. J Neurol Neurosurg Psychiatry 1989;52:1019-1020.

2. Brix G, Semmler W, Port R, Schad LR, Layer G, Lorenz WJ. Pharmacokinetic parameters in CNS Gd-DTPA enhanced MR imaging. J Comput Assist Tomogr 1991;15:621-628.

3. Tofts PS. Modeling tracer kinetics in dynamic Gd-DTPA MR imaging. J Magn Reson Imaging 1997;7:91-101.

4. Landis CS, Li X, Telang FW, et al. Equilibrium transcytolemmal water-exchange kinetics in skeletal muscle in vivo. Magn Reson Med 1999;42:467-478.

5. Landis CS, Li X, Telang FW, et al. Determination of the MRI contrast agent concentration time course in vivo following bolus injection: effect of equilibrium transcytolemmal water exchange. Magn Reson Med 2000;44:563-574.

6. Cao Y, Brown SL, Knight RA, Fenstermacher JD, Ewing JR. The Effect of Intravascular-to-Extravascular Water Exchange on the Determination of Blood-to-Tissue Transfer Constant by MRI. Magn Reson in Med 2005;53:282_0293.

7. Tofts PS, Brix G, Buckley DL, et al. Estimating kinetic parameters from dynamic contrast-enhanced T- weighted MRI of a diffusable tracer: standardized quantities and symbols. J Magn Reson Imaging 1999;10:223-232.

8. Johnson G, Wetzel SG, Cha S, Babb J, Tofts PS. Measuring blood volume and vascular transfer constant from dynamic, $\mathrm{T}(2)^{*}$ weighted contrast-enhanced MRI. Magn Reson Med 2004;51:961968.

9. Li KL, Zhu XP, Waterton J, Jackson A. Improved 3D quantitative mapping of blood volume and endothelial permeability in brain tumors. J Magn Reson Imaging 2000;12:347-357.

10. Vonken EP, van Osch MJ, Bakker CJ, Viergever MA. Simultaneous quantitative cerebral perfusion and Gd-DTPA extravasation measurement with dual-echo dynamic susceptibility contrast MRI. Magn Reson Med 2000;43:820-827.

11. Barbier EL, den Boer JA, Peters AR, Rozeboom AR, Sau J, Bonmartin A. A model of the dual effect of gadopentetate dimeglumine on dynamic brain MR images. J Magn Reson Imaging 1999;10:242253.

12. Weisskoff RW, Boxeman JL, Sorensen AG, Kulke SM, Campbell TA, Rosen BR. Simultaneous Blood Volume and Permeability Mapping Using a Single Gd-Based Contrast Injection. In: Proceedings of the 2nd Annual Meeting of ISMRM; San Francisco, CA, USA, 1994:279.

13. Donahue KM, Krouwer HG, Rand SD, et al. Utility of simultaneously acquired gradient-echo and spin-echo cerebral blood volume and morphology maps in brain tumor patients. Magn Reson Med 2000;43:845-853.

14. Haselhorst R, Kappos L, Bilecen D, et al. Dynamic susceptibility contrast MR imaging of plaque development in multiple sclerosis: application of an extended blood-brain barrier leakage correction. J Magn Reson Imaging 2000;11:495-505.

15. Ostergaard L, Hochberg FH, Rabinov JD, et al. Early changes measured by magnetic resonance imaging in cerebral blood flow, blood volume, and blood-brain barrier permeability following dexamethasone treatment in patients with brain tumors. J Neurosurg 1999; 90:300-305.

16. Rosen BR, Belliveau JW, Aronen HJ, et al. Susceptibility contrast imaging of cerebral blood volume: human experience. Magn Reson Med 1991;22:293-299; discussion 300-293.

17. Cao Y, Shen Z, Chenevert TL, Ewing JR. An alternative approach for estimation of vascular permeability to Gd-DTPA and cerebral blood volume from dynamic T2* weighted contrast-enhanced MRI. In: Proceedings of the 13th Annual Meeting of ISMRM, Miami Beach, FL, USA, 2005. Abstract 2064. 
18. Derdeyn CP, Videen TO, Yundt KD, et al. Variability of cerebral blood volume and oxygen extraction: stages of cerebral haemodynamic impairment revisited. Brain 2002;125(Pt 3):595-607.

19. Ito H, Kanno I, Kato C, et al. Database of normal human cerebral blood flow, cerebral blood volume, cerebral oxygen extraction fraction and cerebral metabolic rate of oxygen measured by positron emission tomography with 150-labelled carbon dioxide or water carbon monoxide and oxygen: a multicentre study in Japan. Eur J Nucl Med Mol Imaging 2004;31:635-643.

20. Rempp KA, Brix G, Wenz F, Becker CR, Guckel F, Lorenz WJ. Quantification of regional cerebral blood flow and volume with dynamic susceptibility contrast-enhanced MR imaging. Radiology 1994; 193:637-641.

21. Ostergaard L, Weisskoff RM, Chesler DA, Gyldensted C, Rosen BR. High resolution measurement of cerebral blood flow using intravascular tracer bolus passages. Part I: Mathematical approach and statistical analysis. Magn Reson Med 1996;36:715-725.
22. Boxerman JL, Hamberg LM, Rosen BR, Weisskoff RM. MR contrast due to intravascular magnetic susceptibility perturbations. Magn Reson Med 1995;34:555-566.

23. Weisskoff RM, Zuo CS, Boxerman JL, Rosen BR. Microscopic susceptibility variation and transverse relaxation: theory and experiment. Magn Reson Med 1994;31:601-610.

24. Press WH, Teukolsky SA, Vetterling WT, Flannery BP. Numerical recipes in C. New York: Cambridge University Press, 1992.

25. Roberts HC, Roberts TP, Brasch RC, Dillon WP. Quantitative measurement of microvascular permeability in human brain tumors achieved using dynamic contrast-enhanced MR imaging: correlation with histologic grade. AJNR Am J Neuroradiol 2000;21:891899.

26. Tofts PS, Cha S, Johnson G. A simple model to characterise bloodbrain barrier leakage from T2*-weighted bolus tracking MRI data. In: Proceedings of the Annual Meeting of ISMRM, Glasgow, Scotland, UK, 2001. Abstract 2242. 